\title{
What is the optimal large airway size reduction value to determine malacia: exploratory bronchoscopic analysis in patients in Mounier-Kuhn syndrome
}

\author{
Evelise Lima, Pedro Rodrigues Genta, Rodrigo Abensur Athanazio, Ascedio José Rodrigues, \\ Maria Aparecida Miyuki Nakamura, Samia Zahi Rached, Eduardo Leite Vieira Costa, Rafael Stelmach \\ Pulmonary Division, Heart Institute (InCor) do Hospital das Clínicas da Faculdade de Medicina da Universidade de São Paulo, São Paulo, Brazil \\ Correspondence to: Evelise Lima. Pulmonary Division, Heart Institute (InCor) do Hospital das Clínicas da Faculdade de Medicina da Universidade de \\ São Paulo, Av. Dr. Enéas Carvalho de Aguiar, 44 - Cerqueira César, São Paulo, SP, Brazil. Email: eveliselima53@gmail.com.
}

Submitted Jul 08, 2020. Accepted for publication Oct 22, 2020.

doi: $10.21037 /$ jtd-20-2395

View this article at: http://dx.doi.org/10.21037/jtd-20-2395

Excessive airway collapse (EAC) has been increasingly identified as a respiratory condition associated with several symptoms and morbidities. However, we observe a huge variability in the way of diagnosing this entity, often using different techniques. We propose a diagnostic method using bronchoscopy (1).

EAC is characterized by trachea and/or bronchi huge collapse, and occurs mainly during expiration $(2,3)$. During expiration occurs an increase in intrathoracic pressure and supporting structures (musculature and cartilage) of central airways, leading to essential balance, which maintains the patency of tracheal and bronchial lumen (4).

Whenever there is pathological involvement of these structures a collapse can be observed (3).

EAC comprises two entities: excessive dynamic airway collapse (EDAC) and tracheomalacia (TM). In EDAC there is an excessive narrowing of the posterior trachea and/or bronchi promoted by membranaceous posterior wall due to weakness of its musculature. TM is characterized by a pathological movement of the entire tracheal structure, involving both muscles and cartilaginous rings. When it extends to the bronchi, it is referred as tracheobronchomalacia (TBM) $(5,6)$.

TM or TBM may affect the airway either diffusely or segmentally $(2,3,5)$. It is classified according to appearance of the trachea in crescent (anteroposterior narrowing), lateral (lateral narrowing) and circumferential (both anteroposterior and lateral narrowing) $(2,3,6)$. Congenital forms manifest predominantly in childhood, and can be related to other respiratory tract conditions, such as Mucopolychondritis or Mounier-Kuhn syndrome (SMK).
Acquired forms are related to traumatic or inflammatory injuries and idiopathic when the cause is unknown $(3,5,6)$.

The recognition of these entities is important since it may be responsible for the existing respiratory symptoms, its worsening or the onset of new symptoms. It may be often confounding with other diseases, such as chronic bronchitis, bronchiectasis, asthma, and chronic obstructive pulmonary disease. The predominant signs and symptoms are cough, abundant tracheobronchial secretion, dyspnea and wheezing (7).

Diagnostic tests include bronchoscopy and computerized tomography scan of the chest (CT). Bronchoscopy is capable of assessing both the anatomical and the dynamic changes of the airway $(2,3,6)$. Classically, the diagnosis is established when there is a $50 \%$ decrease in the tracheobronchial cross sectional area (8), although the use of this parameter remains controversial. The definition of the pathological collapse ranges from $35 \%$ to $80 \%$ of the airway area as shown in studies focused on the definition of pathological collapse (Table 1).

Most studies use chest CT scan for measuring the crosssectional area of the trachea and/or bronchi at maximal inspiration and forced expiration. Some use spirometric monitoring and measured the forced vital capacity (FVC) to ensure maximum expiration. These studies show variations in the normal the values. However, there were different methods and techniques used for measuring, in addition of sample size discrepancies as well as different regions were analyzed.

It is therefore important to define what is really crucial to define excessive collapse. Considering that not all that 


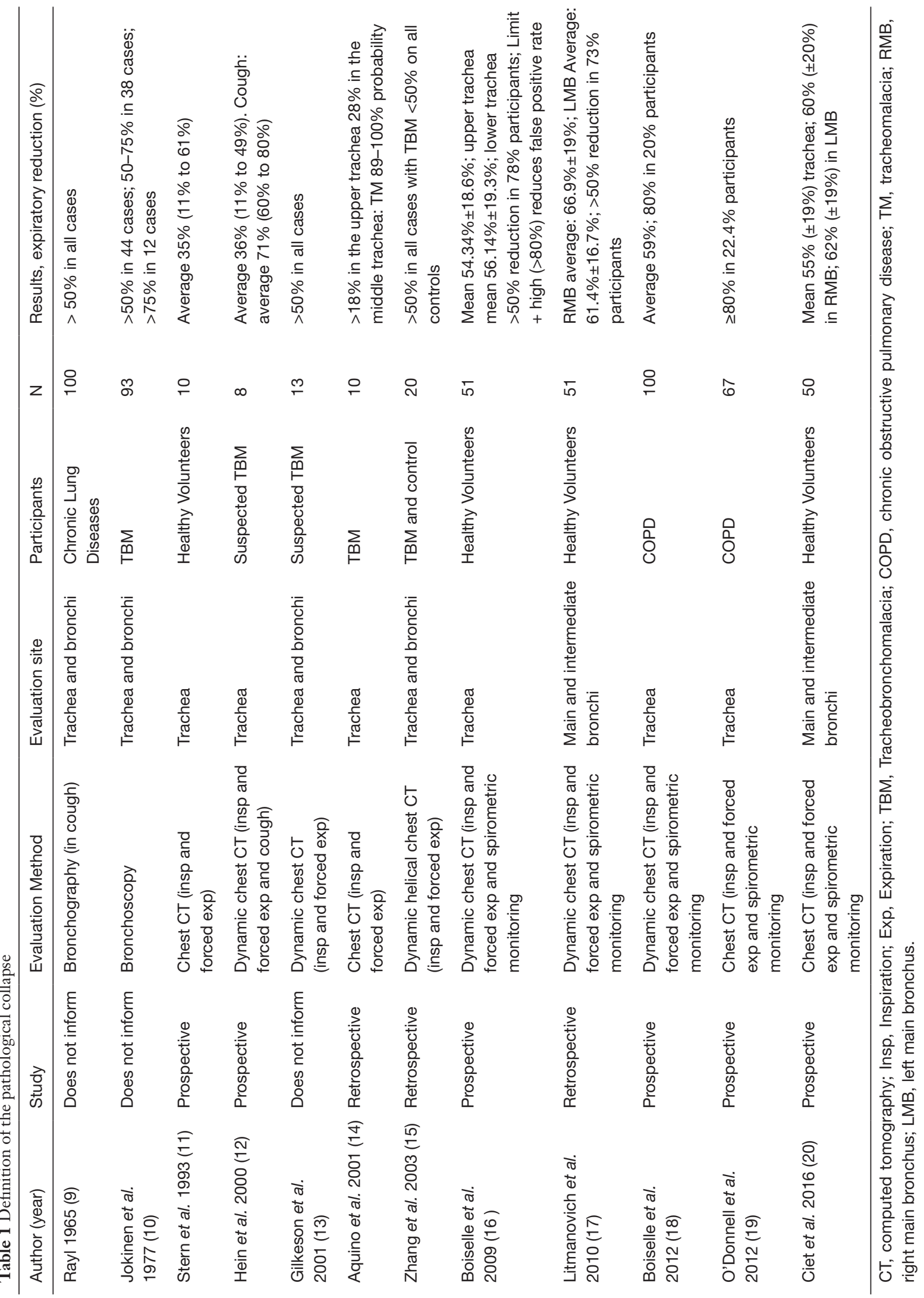



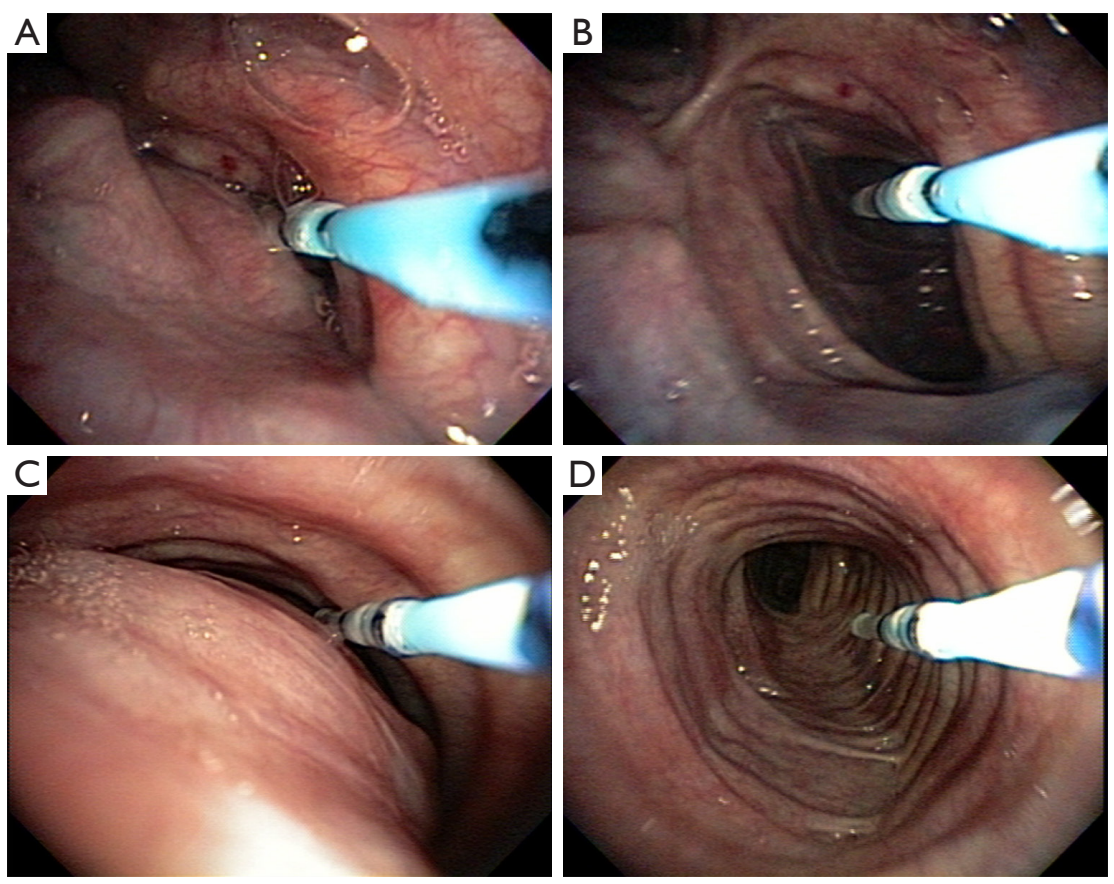

Figure 1 Bronchoscopic view of the trachea, right bronchus and the catheter (blue). (A) Right bronchus during expiration in pressure $0 \mathrm{cmH}_{2} \mathrm{O}$; (B) rigth bronchus during inspiration in pressure $0 \mathrm{cmH}_{2} \mathrm{O} ;(\mathrm{C})$ trachea during expiration in pressure $0 \mathrm{cmH}_{2} \mathrm{O}$; $(\mathrm{D})$ trachea during inspiration in pressure $0 \mathrm{cmH}_{2} \mathrm{O}$.

patients with EAC have symptoms (3), and the fact that healthy individuals may have collapse of up to $80 \%$ on forced expiration (16), the values for the FVC used in chest CT as a diagnostic for pathological collapse remain unanswered.

Another issue is whether the values found during chest CT studies can actually be extrapolated to bronchoscopic analysis, once respiratory cycles are dynamically evaluated. When symptoms are aggravated or triggered by TBM, it has been shown that they may also occur with spontaneous breathing $(2,3,5,6)$. Thus, defining the percentage value of the diagnosis of pathological collapse using findings on forced expiratory chest CT with spirometry monitoring may result in over diagnosis of TBM.

Despite being the gold standard, bronchoscopy $(2,3,6)$ has been underutilized for the analysis of collapse and one of the limiting factors is the lack standardization of the bronchoscopic procedure. Ideally, sedation should be light in a way that the patient can interact with the examiner, but sufficient to avoid discomfort.

Airway size reduction is achieved during spontaneous and forced inspiration and expiration without spirometric monitoring $(2,3)$. Such technical difficulty during the objective analysis yields to results that are dependent on the examiner's judgement $(2,6)$. For an accurate quantitative measurement of the area to be analysed, the examiner is expected to know the distance between the endoscopic lens and the area of interest, keeping the tip of the bronchoscope in the same position, since any change in this distance may result in an increase (nearness) or decrease (distance) from the assessed area (21). In this context, we believe that the best assessment the diagnosis of TBM is via bronchoscopy using a standardized technique with a method that allows objective analysis of the collapse.

In order to find the best way to objectively analyse the variation between inspiration and expiration using bronchoscopy, we designed an assessment protocol to evaluate patients with MKS and TBM (1).

After sedation, we tested the response to continuous positive airway pressure (CPAP) using non-invasive mechanical ventilation (NIMV). A custom catheter was used containing distance markings in centimeters (we used a Fogarty catheter $\mathrm{n}^{\circ} 3$ and made markings in centimeters) that is inserted into the working channel of the bronchoscope. It is kept at a known distance between its distal end and the bronchoscope, minimizing the variation in the distance from the device to the collapse region (Figure 1). NIMV/CPAP titration was performed from 0 to $18 \mathrm{cmH}_{2} \mathrm{O}$ (started in $0 \mathrm{cmH}_{2} \mathrm{O}$ and gradually increases in 
steps by $2 \mathrm{cmH}_{2} \mathrm{O}$ every 10 complete cycles) (1).

The procedure was recorded digitally and the images analysed by a software (Image Processing Toolbox, Matlab®, Natick, MA) capable of evaluating and measuring airway pixel variation by means of comparing the collapse area at $0 \mathrm{cmH}_{2} \mathrm{O}$ with the different titrated pressures (1). The pixels measured at each titration record are proportional to the airway area when corrected for the known distance. From this analysis it was possible to define the best pressure capable to reduce the collapse.

Despite its experimental character, this methodology can be extrapolated to diagnose TBM, allowing a dynamic analysis of the airway that excludes the bias of using FVC in the diagnosis. Therefore, it allows a more realistic analysis of the dynamic variations in airway diameter, thus avoiding an overdiagnosis of pathological collapse, often made in asymptomatic patients.

\section{Acknowledgments}

Funding: None.

\section{Footnote}

Provenance and Peer Review: This article was a free submission to the journal. The article was sent for external peer review.

Conflicts of Interest: All author have completed the ICMJE uniform disclosure form (available at http://dx.doi. org/10.21037/jtd-20-2395). RA Athanazio reports personal fees and other from Astrazeneca, personal fees and other from Vertex, grants, personal fees and other from GSK, personal fees and other from Pfizer, personal fees from Roche, non-financial support from Sanofi, personal fees from Novartis, outside the submitted work. MA Nakamura reports non-financial support from Timpel SA, during the conduct of the study; personal fees from Timpel SA, outside the submitted work. EL Costa reports personal fees from Timpel SA, outside the submitted work. R Stelmach reports grants from São Paulo Research Foundation, grants and personal fees from Novartis, grants, personal fees and nonfinancial support from AstraZeneca, grants from MSD, grants, personal fees and non-financial support from Chiesi, personal fees and non-financial support from Boheringer Ingelheim , outside the submitted work. The other authors have no conflicts of interest to declare.
Ethical Statement: The authors are accountable for all aspects of the work in ensuring that questions related to the accuracy or integrity of any part of the work are appropriately investigated and resolved.

Open Access Statement: This is an Open Access article distributed in accordance with the Creative Commons Attribution-NonCommercial-NoDerivs 4.0 International License (CC BY-NC-ND 4.0), which permits the noncommercial replication and distribution of the article with the strict proviso that no changes or edits are made and the original work is properly cited (including links to both the formal publication through the relevant DOI and the license). See: https://creativecommons.org/licenses/by-nc-nd/4.0/.

\section{References}

1. Lima E, Nakamura MAM, Genta PR, et al. Improving Airways Patency and Ventilation Through Optimal Positive Pressure Identified by Noninvasive Mechanical Ventilation Titration in Mounier-Kuhn Syndrome: Protocol for an Interventional, Open-Label, Single-Arm Clinical Trial. JMIR Res Protoc 2020;9:e14786.

2. Buitrago DH, Wilson JL, Parikh M, et al. Current concepts in severe adult tracheobronchomalacia: evaluation and treatment. J Thorac Dis 2017;9:E57-66.

3. Hammond K, Ghori UK, Musani AI. Tracheobronchomalacia and Excessive Dynamic Airway Collapse. Clin Chest Med 2018;39:223-8.

4. Levitzky MG. Mechanics of Breathing. In: Pulmonary Physiology. Seventh Edition. New York: McGrawHill, 2007:11-53.

5. Murgu S, Colt H. Tracheobronchomalacia and excessive dynamic airway collapse. Clin Chest Med 2013;34:527-55.

6. Murgu SD, Colt HG. Description of a multidimensional classification system for patients with expiratory central airway collapse. Respirology 2007;12:543-50.

7. Krustins E, Kravale Z, Buls A. Mounier-Kuhn syndrome or congenital tracheobronchomegaly: a literature review. Respir Med 2013;107:1822-8.

8. Carden KA, Boiselle PM, Waltz DA, et al. Tracheomalacia and tracheobronchomalacia in children and adults: an indepth review. Chest 2005;127:984-1005.

9. Rayl JE. Tracheobronchial collapse during cough. Radiology 1965;85:87-92.

10. Jokinen K, Palva T, Sutinen S, et al. Acquired tracheobronchomalacia. Ann Clin Res 1977;9:52-7. 
11. Stern EJ, Graham CM, Webb WR, et al. Normal trachea during forced expiration: dynamic CT measurements. Radiology 1993;187:27-31.

12. Hein E, Rogalla P, Hentschel C, et al. Dynamic and quantitative assessment of tracheomalacia by electron beam tomography: correlation with clinical symptoms and bronchoscopy. J Comput Assist Tomogr 2000;24:247-52.

13. Gilkeson RC, Ciancibello LM, Hejal RB, et al. Tracheobronchomalacia: dynamic airway evaluation with multidetector CT. AJR Am J Roentgenol 2001;176:205-10.

14. Aquino SL, Shepard JA, Ginns LC, et al. Acquired tracheomalacia: detection by expiratory CT scan. J Comput Assist Tomogr 2001;25:394-9.

15. Zhang J, Hasegawa I, Feller-Kopman D, et al. 2003 AUR Memorial Award. Dynamic expiratory volumetric CT imaging of the central airways: comparison of standard-dose and low-dose techniques. Acad Radiol 2003;10:719-24.

16. Boiselle PM, O'Donnell CR, Bankier AA, et al. Tracheal collapsibility in healthy volunteers during forced

Cite this article as: Lima E, Genta PR, Athanazio RA, Rodrigues AJ, Nakamura MAM, Rached SZ, Costa ELV, Stelmach R. What is the optimal large airway size reduction value to determine malacia: ex-ploratory bronchoscopic analysis in patients in Mounier-Kuhn syndrome. J Thorac Dis 2021;13(1):425-429. doi: 10.21037/jtd-20-2395 expiration: assessment with multidetector CT. Radiology 2009;252:255-62.

17. Litmanovich D, O'Donnell CR, Bankier AA, et al. Bronchial collapsibility at forced expiration in healthy volunteers: assessment with multidetector CT. Radiology 2010;257:560-7.

18. Boiselle PM, Michaud G, Roberts DH, et al. Dynamic expiratory tracheal collapse in COPD: correlation with clinical and physiologic parameters. Chest 2012;142:1539-44.

19. O'Donnell CR, Litmanovich D, Loring SH, et al. Age and sex dependence of forced expiratory central airway collapse in healthy volunteers. Chest 2012;142:168-74.

20. Ciet P, Boiselle PM, Michaud G, et al. Optimal imaging protocol for measuring dynamic expiratory collapse of the central airways. Clin Radiol 2016;71:e49-55.

21. Ferguson GT, Benoist J. Nasal continuous positive airway pressure in the treatment of tracheobronchomalacia. Am Rev Respir Dis 1993;147:457-61. 